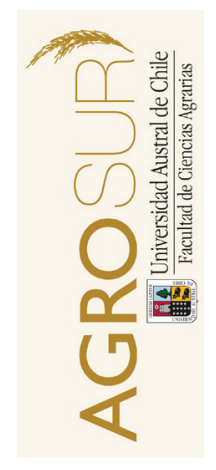

\title{
Construyendo mercados desde la propia finca. Tres experiencias en la agricultura familiar
}

\section{Developing markets from the farm itself: three experiences of family farming}

\author{
Rodríguez-Sperat, R. ${ }^{a *}$, Paz, R. ${ }^{b}$, Suárez, V. , Díaz, J.P. ${ }^{d}$ \\ ${ }^{a}$ Investigador del CONICET/INDES. Catamarca 350. Santiago del Estero. \\ ${ }^{b}$ Investigador del CONICET/INDES. Mendoza 496. Santiago del Estero.
}

${ }^{c}$ Becaria del CONICET Tipo I Temas Estratégicos. INDES/UNSE. Av. Belgrano (S) 2180. Santiago del Estero.

${ }^{d}$ Docente en la Cátedra de Extensión y Desarrollo Rural. FAYA/UNSE. Av. Belgrano (S) 1912. Santiago del Estero.

\begin{tabular}{l} 
A R T I C L E I N F O \\
\hline Article history: \\
Received 06.11.14 \\
Accepted 13.05.15 \\
\hline Keywords: \\
Family farming \\
Productive strategies \\
Short food circuits \\
Value added \\
Food sovereignty \\
\hline Case Study, \\
Agriculture Economics and \\
Rural Development \\
\hline *Corresponding author: \\
Ramiro Rodríguez-Sperat \\
E-mail address: \\
ramirorodriguezsperat@ \\
hotmail.com
\end{tabular}

\begin{abstract}
A B S T R A C T
There is widespread consensus on the need to strengthen family farming in order to guarantee the global food sovereignty. One of the biggest challenges in achieving this goal is that most part of the food is traded through markets handling patterns that are hardly compatible with family farming. Perhaps the key to solving this problem lies in identifying the achievements and paths followed by the same producers that have developed alternative marketing strategies. Many family farmers have developed viable entrepreneurships, building innovative markets for their products based on short circuit commercialization systems that not only increase profitability due to a cut of intermediaries, but also lower the food prices and provides the final consumer with better quality products as a consequence of the added value strategy followed by the producers. In that context, this study exposes three cases of family farms located in the irrigation area of Río Dulce, Province of Santiago del Estero (Argentina). The cases present a similar productive, economic and social logic, characterized by a strong co-production between man and nature, provided by a combination of endogenous resources, value added, generation of technological novelties and craftsmanship, among others, within a strong process of family labor intensification.
\end{abstract}

\section{RESUMEN}

Existe consenso sobre la necesidad de potenciar la agricultura familiar en pos de garantizar la soberanía alimentaria global; pero uno de los mayores desafíos para lograr este objetivo es el hecho de que la mayor parte de los alimentos es comercializada a través de mercados que manejan pautas poco compatibles con esta forma de producir. Tal vez las claves para solucionar este problema se encuentren en identificar los logros y caminos llevados adelante por los mismos productores que han desarrollado estrategias alternativas de mercadeo y, a partir de allí, buscar la forma de potenciarlos desde las políticas públicas. Muchos agricultores familiares han logrado ser viables en sus emprendimientos, construyendo nuevos mercados sobre la base de sistemas de comercialización asentados en circuitos cortos, que no solo aumentan su rentabilidad por la reducción de intermediarios, sino que a su vez disminuyen el precio de los alimentos y ofrecen productos de mayor calidad al consumidor final como consecuencia de su estrategia de agregado de valor. El presente trabajo se desarrollará en esa línea, presentando tres casos de agricultura familiar ubicados en el área de riego de la provincia de Santiago del Estero. Las explotaciones presentadas tienen una lógica productiva, económica y social similar, caracterizada por una fuerte coproducción hombre-naturaleza, la vinculación entre los recursos de la propia explotación, el agregado de valor, la generación de novedades tecnológicas y la artesanalidad, entre otros, en el marco de un fuerte proceso de intensificación de la mano de obra familiar.

Palabras clave: Agricultura familiar, estrategias productivas, circuitos cortos, agregado de valor, soberanía alimentaria

\section{INTRODUCCIÓN}

El escenario de la producción y comercialización de alimentos ha cambiado sustancialmente en los últimos 30 años. La emergencia de nuevos circuitos financieros, tecnologías productivas y formatos comerciales han re- volucionado los mercados alimentarios. En la actualidad, los productos agrícolas de distintas partes del mundo son trasladados diariamente entre los diferentes países y continentes, compitiendo en los mercados de destino con las producciones locales, bajo distintas combinaciones de precios y calidad (Barbosa-Calvacanti, 2004). 
Sin embargo, este mismo período de tiempo también se ha caracterizado por la volatilidad y un aumento generalizado en el precio de los alimentos -especialmente a partir del 2007-, dejando evidencias de que el sistema de producción, distribución y comercialización de alimentos que predomina en la actualidad dista mucho de ser el ideal, y llevando a los gobiernos a un consenso sobre la necesidad de potenciar la agricultura familiar como elemento clave para garantizar la Seguridad Alimentaria ${ }^{1}$ global (CEPAL, 2013a).

Uno de los interrogantes que surge en este contexto se encuentra relacionado con la viabilidad de articular la agricultura familiar con el sistema comercial actualmente establecido. La alta concentración observada tanto en la producción como en la distribución de los alimentos $^{2}$ (Jacoby, 2013), el rol de los supermercados como principales centros de abastecimiento para la población (Rodríguez, 2013) y la distancia cada vez mayor entre la localización física de la producción y los consumidores $^{3}$ (que lleva a que gran parte de la población desconozca el origen de los alimentos que consume), son algunas de las mayores críticas al sistema agroalimentario actual.

Desde otra perspectiva y pensando en las posibilidades de desarrollo de la pequeña producción, parecería más apropiado buscar y consolidar circuitos alternativos de comercialización, más cortos y directos que no se contrapongan con los valores de la agricultura familiar, que busca promover alternativas más equitativas y sustentables -como las ferias francas y campesinas, las redes de comercio justo y las de agricultores urbanos-, donde el estilo de vida del agricultor familiar y su forma de producir se hacen presentes (INTA, 2010; Van der Ploeg, 2010; Paz et al., 2013; Rodríguez-Sperat et al., 2014).

La agricultura familiar se construye y reproduce mayormente a través de circuitos cortos y descentra-

El concepto de seguridad alimentaria surge en la década del 70. En la actualidad, existe una definición oficializada por la Organización de las Naciones Unidas para la Agricultura y la Alimentación (FAO) durante la Cumbre Mundial de la Alimentación (1996). La definición adoptada indica que existe seguridad alimentaria "Cuando todas las personas tienen en todo momento acceso físico, social y económico a los alimentos suficientes, inocuos y nutritivos que satisfagan sus necesidades energéticas diarias y preferencias alimentarias para llevar una vida sana y activa".

2 En la actualidad, por ejemplo, entre Nestlé, Kraft Food's y General Mills se reparten cerca del $40 \%$ del mercado mundial de los productos procesados (porcentaje que se incrementa al $70 \%$ si solo se toman los alimentos que se venden en Estados Unidos), y entre Coca-Cola y Pepsi-Cola manejan el $50 \%$ del mercado mundial de bebidas gaseosas (Jacoby, 2013).

3 Por solo ilustrar esta realidad con algunos números, en una encuesta realizada en Brasil en 2011, se registró que el $80 \%$ de las compras de hortalizas se realizan en supermercados y sólo un $8 \%$ en ferias (Andrade de Almeida Cunha, 2013). Para el caso de Argentina, existen estimaciones de que entre solo 7 cadenas de supermercados tienen la capacidad de vender el $58 \%$ del total alimentos y bebidas del país, y que de ese porcentaje el 70\% lo representan sólo Carrefour, Cencosud y Coto (FAECyS, 2012). Incluso se sostiene que un fenómeno muy parecido se ha dado casi simultáneamente en el resto de los países de América Latina, protagonizado por las mismas cadenas internacionales (Chiodo, 2010). lizados, que vinculan a la producción y al consumo de alimentos, y más en general, a la agricultura y a la sociedad en su conjunto. Así se genera una forma de comercio basada en la venta directa de productos frescos o de temporada, que reduce al mínimo la intermediación, aproxima más a los productores con los consumidores, fomenta el trato humano, y genera un impacto medioambiental más bajo, al no transportar los productos a largas distancias ni envasarlos (CEPAL, 2013b).

Al repasar la bibliografía, se observa que ha existido una preocupación conceptual y metodológica vinculada con la temática. Algunos autores europeos hablan de las cadenas cortas de comercialización, y sostienen que las mismas surgen como consecuencia de cambios en los eslabones de la cadena agroalimentaria: del lado del consumidor, por la desconfianza que genera la agricultura tradicional en relación a la calidad de los alimentos que produce (Marsden, 1998), y del lado de los productores, por la presión sobre los precios, el incremento de los costos de producción y consecuentemente, la reducción de los márgenes económicos generados como consecuencia de la desregulación de los mercados (Renting et al., 2003).

Otros autores sostienen que deberían hacerse algunas reconsideraciones en la literatura sobre los circuitos cortos (o circuitos alternativos), ya que la misma no toma en cuenta la noción de "lugares de mercado" (Shanin, 1973). Aducen que se habla de circuitos, que llevan productos o servicios desde el productor hacia el consumidor, a través de la distancia social y/o geográfica más corta posible. Pero este análisis por lo general pierde de vista el hecho de que dichos circuitos son partes intrínsecas de un nuevo mercado. Lo mismo ocurre con la noción de "alternativo", que tiende a referirse solamente a la cuestión normativa (Hebinck et al., 2015). En este marco, se sugiere que resultaría apropiado abordar el tema desde una perspectiva más amplia, utilizando el concepto de "mercados anidados" (Van der Ploeg, 2015)

También, y ya pasando a un plano más regional, existen en Latinoamérica algunos estudios que abordan la temática desde el análisis de la oferta del producto, donde consideran distintas alternativas para reducir intermediarios en la comercialización de los alimentos (Chauveau y Taipe, 2012; VSF-CICDA, 2013; Aubron et al., 2013; Lacroix y Cheng, 2014).

Desde nuestra perspectiva, existe una dimensión adicional que debería ser considerada para comprender de una manera más holística los circuitos cortos de comercialización, y que hasta el momento no ha sido tan abordada en la literatura específica. Esta dimensión se relaciona con la propia lógica o estilo de producción sobre el cual se sustenta el proceso productivo, y que en definitiva lleva a una explotación a adoptar este tipo de estrategia comercial. Consideramos que incorporar esta perspectiva es central, y sobre todo cobra mayor relevan- 
cia cuando se analiza dicho proceso en un actor agrario tan complejo como el campesino o el agricultor familiar.

Tal vez las claves para una mejor aproximación a los circuitos cortos en la agricultura familiar, se encuentren en identificar los logros y caminos llevados adelante por los mismos productores que han desarrollado estrategias de mercadeo alternativas al sistema dominante, y buscar, a partir de allí, la forma de potenciarlos desde las políticas públicas.

Precisamente este artículo tiene por objetivo identificar las lógicas y dinámicas que se esconden detrás de los circuitos cortos, desde el estudio de caso de tres agricultores familiares situados en el área de riego de la provincia de Santiago del Estero, Argentina. Estos productores tienen la particularidad de haber logrado dotar de una cierta viabilidad a sus emprendimientos productivos, construyendo nuevos mercados para sus productos sobre la base de un sistema de comercialización asentado en circuitos cortos, pero sin descuidar la relación con su estilo de producción.

\section{Construcción de los circuitos cortos en la agricultura familiar}

Antes de analizar los tres casos, se hace necesario establecer algunas pautas conceptuales en relación a los circuitos cortos y a la condición del contexto en donde se desarrolla la agricultura familiar. La agricultura familiar desarrolla sus actividades cotidianas en un contexto caracterizado por relaciones de dependencia, la mayoría de las veces asimétrica, donde existe un tenue pero constante proceso de transferencia de riqueza que se materializa principalmente en la relación con los mercados formales (Schejtman, 1981; Van der Ploeg, 2010).

La construcción de los circuitos cortos llevada adelante por los agricultores familiares no es otra cosa que una forma de resistencia a su propia condición de marginalidad. Es la construcción de otro tipo de articulación con los mercados, donde tales formas de relacionarse presentan una mayor equidad, flexibilidad, movilidad y autonomía (VSF-CICDA, 2013; Paz y Bruno, 2013).

Por otra parte es importante destacar que los circuitos cortos no pueden ser interpretados desde una perspectiva analítica y puntual, como tampoco desde la condición de hostilidad por parte de los canales convencionales de comercialización. Los circuitos cortos constituyen una estrategia de resistencia, que se sostiene y gana identidad a partir de otras condiciones que son propias de la agricultura familiar. La intensificación de la mano de obra familiar tanto en los procesos de gestión como en los de producción, los procesos de no mercantilización en especial de los factores productivos, la artesanalidad y el saber hacer orientados al agregado de valor, volúmenes de producción que resultan manejables, flexibles y en sintonía con los mercados que vienen construyendo, conforman las dimensiones centrales que dan identidad y sostienen a la construcción de los circuitos cortos (Paz et al., 2011).

Es desde esta perspectiva que se presentarán tres estudios de caso donde se buscará una representación general de estas diferentes dimensiones y su relación con los circuitos cortos.

\section{MATERIAL Y MÉTODOS}

El enfoque de sistemas de producción (Berdegué y Nazif, 1988) es una aproximación que permite comprender la composición, funcionamiento y dinámica de las unidades productivas que construyen los productores, a partir del despliegue de una diversidad de estrategias no sólo productivas, sino también económicas, sociales y de relación con el medio ambiente que lo rodea. La mayor contribución de este abordaje reside, quizás, en la posibilidad de complementar análisis duros agronómicos y económicos con un análisis más de tipo social, permitiendo captar también la percepción del productor mismo, es decir entender aquellas características culturales que influyen en la toma de decisiones (Berdegué y Nazif, 1988).

Es así que se consideró conveniente realizar el abordaje de los casos en dos etapas: la primera mediante un cuestionario semi-estructurado con preguntas cerradas y solo algunas preguntas abiertas, buscando captar las estrategias productivas y reproductivas a partir de dimensiones técnico-productivas y sociales (tales como estructura demográfica y ocupación de la familia, diversificación del sistema agropecuario, condición de los recursos, diagrama de producción, diagrama de circulación, ingresos parciales y totales, entre otros).

La segunda etapa surge a partir de la identificación de aquella actividad productiva específica a estudiar con mayor nivel de detalle (la producción de pollos, de quesos de cabra y de chacinados respectivamente), donde se realizaron entrevistas en profundidad a los actores involucrados, observación directa del proceso productivo y el seguimiento de las actividades a partir de planillas diseñadas para tal efecto. Esta segunda etapa tuvo como objetivo comprender con mayor profundidad la lógica y dinámica de los estilos de producción, así como la trayectoria y el desarrollo de la estrategia comercial.

Para evitar caer en diseños de investigación estructurados y teniendo en cuenta que el objetivo de la investigación se extiende más allá de una descripción, es decir que se apunta a comprender en terreno las estrategias de producción y comercialización así como las relaciones que éstas suponen, es que se siguió un diseño flexible para complementar los datos obtenidos de instancias cuantitativas con técnicas de observación y análisis más cualitativas (Vasilachis de Gialdino, 2006).

Los casos estudiados vienen siendo trabajados por los autores de este artículo desde hace varios años, en 
distintas instancias institucionales a través de proyectos de asistencia técnica, lo que facilitó el dialogo entre productores e investigadores generando un contexto de confianza y muy buena disposición. Sin embargo, y a los fines de hacer comparativos los datos, se buscó seleccionar para este trabajo un período temporal de al menos un ciclo productivo.

Es también a partir de este trabajo previo que se seleccionaron los tres casos de estudio, teniendo en cuenta los siguientes criterios. Se buscó que las explotaciones: a) mantengan un estilo de producción asentado sobre estrategias relacionadas con la agricultura familiar; b) hayan construido una estrategia de venta directa, con una demanda del producto reconocida entre los consumidores por la calidad del mismo; y c) correspondan a emprendimientos productivos viables, que hayan logrado sostenerse en el tiempo y que tengan cierta proyección futura.

\section{DESARROLLO}

Los casos de agricultura familiar que se presentan a continuación se encuentran ubicados en el área de riego de la provincia de Santiago del Estero. Esta zona se compone por los departamentos Capital, Banda, Silípica y Robles, y dentro de la zonificación productiva realizada por la provincia, fue denominada como área de alta densidad poblacional y crecimiento económico, constituyendo una de las áreas más fértiles de la misma (Rodríguez-Sperat y Peiretti, 2007).

El primer caso está dado por la explotación de Don Ricardo, quien desde hace un poco más de 2 años ha incorporado a su sistema productivo el engorde de pollos de campo, como una actividad adicional a su sistema productivo diversificado. El segundo, lo representa la explotación de Don Francisco, que cuenta con una producción diversificada y un tambo-fabrica que le permite producir más de $2.000 \mathrm{~kg}$ de queso de cabra por año. La producción de quesos fue instalada en la explotación desde hace más de 12 años. Finalmente el tercer caso se denomina Colonia Jaime, un emprendimiento agropecuario de 600 hectáreas cuya particularidad es el manejo productivo y social de manera comunitaria, donde viven más de 80 personas ( 25 familias). Este caso será analizado desde la producción de chacinados de cerdo, actividad que viene siendo desarrollada por la colonia desde hace más de 30 años.

Los tres casos se encuentran transitando distintas etapas del proceso de construcción de una identidad productiva y comercial, siendo el primero relativamente nuevo en la actividad (comenzó con los pollos a fines del año 2011, y se hizo cargo de la granja a fines del 2009), el segundo con una experiencia de más de 17 años en el rubro y el tercero funciona desde 1932, disponiendo de mayores antecedentes productivos y mercados más consolidados.

\section{Caso 1: El sistema de producción de Don Ricardo.}

La explotación estudiada se encuentra ubicada en Villa Robles, sobre la Ruta Provincial № 1 a $32 \mathrm{~km}$ de la capital de la provincia de Santiago del Estero. El predio ha sido trabajado por la familia a lo largo de varias generaciones y comprende alrededor de 8 hectáreas, todas ellas de muy buena aptitud para el cultivo. De la extensión total, se cultivan aproximadamente 7,5 hectáreas ( 5 de alfalfa, 1,5 de maíz y 1 de cebada) y el resto se ocupa para la vivienda familiar, un galpón y la cría de animales.

La estructura familiar está compuesta por Don Ricardo (49 años), quien es el jefe de la explotación desde el 2009 -año en el que se hizo cargo del predio por la enfermedad de su padre-, su pareja Arminda (42 años), su hija Luciana (de solo algunos meses de edad), su madrastra Doña Nora (57 años) y su sobrina Johana (5 años).

Don Ricardo interviene directamente tanto en el proceso productivo como en el de gestión de la explotación. Hasta principios del 2013 Arminda y en menor medida Doña Nora lo ayudaban en todas las tareas productivas del campo, y además se encargaban del manejo de la casa y de administrar el dinero que ingresaba en la explotación (hasta entonces la mano de obra era casi $100 \%$ familiar). Pero a partir del embarazo de Arminda empezaron a delegar algunas cuestiones productivas, ocupándose más de las tareas domésticas, el cuidado de la huerta familiar y la elaboración de dulces y escabeches caseros ${ }^{4}$.

A partir de ese momento se aumentó la contratación a peones de la zona para que ayuden en tareas específicas en las diferentes producciones (siembras, cosechas, enfardado, pariciones, faenas y tareas de mantenimiento en general), pero siempre manteniendo una participación activa y sin delegar las tareas de gestión. No se trata de empleados fijos sino que los contrata para estas tareas en particular, pagándoles por hora y/o día trabajado.

Estos aspectos sobre la dinámica de la mano de obra son comentados debido a que resultan de suma trascendencia para este tipo de agricultura, por cuanto constituyen uno de los pilares sobre los que se asienta el sistema productivo y reproductivo, y contribuyen a garantizar la continuidad de la explotación en el tiempo. En este caso, la reasignación de tareas demuestra la flexibilidad de la explotación para adaptarse a contextos o situaciones cambiantes; en términos de Van der Ploeg (2013), la capacidad para "danzar en el tiempo".

En relación a la dimensión productiva, presenta una gran diversidad con distintas actividades productivas. Las actividades principales de la explotación son el en-

\footnotetext{
Sin embargo las mujeres siguen jugando un rol muy importante en la gestión de la explotación, compartiendo la toma de decisiones e incluso proporcionando su mano de obra en determinadas procesos donde se necesita de una mayor artesanalidad (como la faena, limpieza y embolsado de los pollos, la limpieza de los corrales, etc.)
} 
gorde de aves de corral, la venta de fardos de alfalfa y la cría y engorde de porcinos (producciones que se realizan durante todo el año).

En el engorde de pollos se percibe una fuerte lógica de mercado: casi la totalidad de la producción es vendida y la mayoría de los elementos de trabajo y los otros recursos productivos (salvo la mano de obra y parte del maíz que utiliza como suplemento en la alimentación) son movilizados a través de los respectivos mercados de insumos. Por año se engordan aproximadamente 800 pollitos, que llegan a su peso óptimo en 3,4 kg (2,6 kg limpios y faenados) entre los 45 y 60 días de alimentación. La producción es continua durante todo el año, utilizando dos corrales para las diferentes tandas de pollitos que va introduciendo en intervalos de 40 días aproximadamente.

La actividad de cría y engorde de porcinos también se realiza con el objetivo de producción hacia el mercado, pero en esta actividad -salvo en la alimentación de los lechones- no se observa una gran externalización de los recursos productivos que se utilizan: los corrales y las parideras se realizan en forma artesanal, con restos de madera y pallets que hay en el campo, las madres pastorean en un lote de alfalfa, la sanidad y las pariciones las maneja el mismo productor. Se trata de un sistema semi-intensivo de cría y engorde de cerdos, pero que es complementado con el pastoreo y encierro en corrales y parideras.

Es especialmente en estas dos actividades donde Don Ricardo busca obtener un mayor valor agregado en su producción, apuntando a conseguir mejores precios en base a una diferenciación en la calidad de sus productos (sobre todo en el engorde de pollos de campo).

La producción de alfalfa es la actividad que ocupa más superficie de la explotación (de las 7,5 hectáreas cultivables, se destinan 5 para la actividad). La producción está orientada principalmente al mercado (el 70\% de los fardos se venden), pero también cumple otro rol muy importante en la explotación ya que es utilizada para la alimentación de los animales del predio, función a la que se destinan no solo el $30 \%$ restante de los fardos, sino también la alfalfa de menor calidad, que no llega a ser enfardada y es almacenada en parvas a la intemperie y cubierta con plástico para su conservación hasta su posterior utilización.

Estas actividades se complementan con la siembra de maíz y cebada (como actividades secundarias), algunos bovinos, la cosecha de frutales para la elaboración de dulces caseros, una pequeña huerta y algunos animales de corral (gallinas, pavos, conejos), que mayormente son para el autoconsumo, pero también se utilizan en la elaboración de escabeches.

Especialmente la producción de dulces y escabeches es una nueva apuesta de la familia, y en esta actividad también se percibe la búsqueda de valor agregado en base a la calidad del producto, pero recién están dando sus primeros pasos en ella y aún es muy incipiente como para ser analizada en mayor profundidad.

\section{Construyendo un mercado para el pollo de campo.}

El productor estudiado ha desarrollado un esquema comercial que, de acuerdo a la clasificación de la CEPAL para los circuitos de proximidad, es el resultado de la combinación de distintos formatos: la venta directa en la explotación, la venta directa en ferias locales, el reparto a domicilio y la venta por correspondencia (Internet, otros) (CEPAL, 2013b).

Don Ricardo ha logrado ir creciendo en estos canales fundamentalmente a través del "boca a boca", incrementando su clientela en base a su prestigio como buen agricultor, percepción que reafirma a simple vista mediante la oferta de un pollo de mayor tamaño que el promedio, de un tono amarillento característico de la alimentación con maíz y un sabor muy particular, elementos que agregan valor al producto desde el punto de vista de la percepción del cliente, permitiéndole al productor diferenciarse de la competencia (Porter, 1985) y obtener un mayor precio de venta.

Una de las grandes interrogantes que existen en la literatura sobre la conveniencia de orientar la pequeña producción familiar hacia el mercado, radica en si este proceso trae aparejado la destrucción de la "autonomía" de la explotación, en el sentido de que el capital y las instituciones externas penetran en la granja, y gradualmente van tomando el control sobre el proceso productivo y las decisiones (Long, 1986). Al respecto, en sus primeras experiencias, Don Ricardo intentó colocar su producción en circuitos tradicionales consolidados (almacenes, carnicerías, etc.), pero ante las condiciones de cantidades, plazos, tamaños y precios que le exigían, rápidamente identificó que para seguir manteniendo un esquema de producción que no comprometa su autonomía, su venta necesariamente debería ser más directa.

Fue de esa manera como comenzó el proceso de construcción de un esquema comercial basado en la venta directa sin intermediarios, apoyándose fuertemente en sus vínculos sociales para promocionar sus productos. En ese sentido, Gavin Smith comenta que este tipo de explotaciones obtiene "...buena parte de su ventaja competitiva por el uso que hace de las relaciones sociales en las que se halla inmersa..." (Smith, 1985: pág. 99), y este accionar se observa claramente en el caso estudiado: los primeros puntos donde logró colocar su producción fueron los vecinos y conocidos de la zona, los compañeros de trabajo de una de sus hermanas que trabaja en un sanatorio de la ciudad, los antiguos clientes que tenía la explotación y la gente que llegaba al predio por otros asuntos.

Los técnicos de la Subsecretaria de Agricultura Familiar de la Nación ${ }^{5}$ también se convirtieron en una fuente

Institución que asiste al productor desde que se hizo cargo de la explotación, brindándole asistencia técnico-productiva, legal y financiera. 
importante de nuevos clientes, ayudando a promocionar el producto no solo entre otros técnicos de la institución, sino también entre sus allegados. La estrategia comercial desplegada por Don Ricardo se complementó con la asistencia a distintas ferias provinciales para difundir sus productos y así obtener nuevos clientes.

Otra cuestión interesante de analizar es la forma en la que el productor fue incorporando paulatinamente los avances en materia de telecomunicaciones y redes sociales. A fines del 2012 se registró en Facebook y Whatsapp y, mediante un teléfono celular, comenzó a promocionar y coordinar sus ventas también por esa vía, sacando fotos a sus productos y etiquetando en ellas a todos sus contactos.

Este hecho representó una bisagra en su esquema comercial, ya que le permitió sortear en gran medida el obstáculo de la distancia geográfica con la ciudad (Midoux, 2013), al menos desde un plano "virtual", facilitándole las acciones de promoción y venta directa, sin la necesidad de depender de una persona que le levante los pedidos (función que previamente desempeñaba su hermana y algunos técnicos de la Subsecretaría).

Actualmente sus canales de venta ya están más consolidados, los clientes directamente acuden a su finca, o le compran por teléfono, Whatsapp y/o Facebook. De esta forma, cuando viaja a la ciudad, carga conservadoras con pollos y entrega la mercadería a los clientes que le hicieron encargos, y además visita determinados lugares donde habitualmente le compran cuando lo ven (hospitales, dependencias gubernamentales, etc.).

Don Ricardo sostiene que por el momento prefiere no recurrir a intermediarios porque esa modalidad comercial le requeriría de tener más corrales y producir una mayor cantidad de pollos. Comenta por ejemplo que hace poco tiempo un amigo que tiene carnicerías, le pidió que le venda pollos, pero para abastecerlo necesitaría contar con 4 corrales (actualmente tiene solo 2 ), ya que le pide que le garantice una cantidad importante de pollos por semana, y esto además lo dejaría sin producción para sus clientes habituales.

En su conjunto, todas estas estrategias constituyen parte de un proceso más amplio, que da cuenta de las respuestas activas de los agricultores familiares a la creciente mercantilización, permitiendo que el mercado y otras fuerzas "externas" ingresen en el mundo de la explotación familiar, integrándolos dentro de sus propias estrategias agrícolas, pero al mismo tiempo cuidando de mantener un grado relativo de autonomía en materia de toma de decisiones (Van der Ploeg, 2010).

El caso de Don Ricardo demuestra que lograr esta "autonomía relativa" es posible, ya que mediante decisiones comerciales concretas como por ejemplo, de no ceder ante las imposiciones de determinados circuitos comerciales, el productor continúa controlando la organización de su propio trabajo y mantiene sus estrategias de no mercantilización (Paz, 2008). De esta forma, se podría sostener que la actual fortaleza y viabilidad de la producción familiar no descansa en su grado de orientación al mercado, sino en la forma en la que el productor logra ir encontrando los circuitos comerciales apropiados para no comprometer la autonomía en su producción.

\section{El proceso de ganar mercado ajustando la producción.}

A pesar de la importancia relativa en términos monetarios que esta actividad tiene para la familia, el engorde de pollitos es algo relativamente novedoso en la explotación estudiada.

El productor se inició en esta producción hacia fines del año 2011, mediante un proyecto de capacitación y financiamiento impulsado desde la Subsecretaría de Desarrollo Rural y Agricultura Familiar de la Nación. La idea original y diseño posterior del proyecto surgieron de un técnico de dicha institución, y no era una práctica habitual de la zona ni tampoco de la familia, en lo que podría caracterizarse como un típico proceso de transferencia y adopción de tecnologías procedentes del exterior de las unidades de producción o "exotecnologías" (Cáceres et al., 1997).

En el Cuadro 1 se puede observar la evolución en términos de cantidades de pollitos incorporados al engorde. En forma paralela, a partir del inicio de esta actividad, se observa un claro proceso de experimentación y adaptación tecnológica por parte del productor, de menor o mayor intensidad de acuerdo a los resultados que iba obteniendo, con una notable capacidad de innovación para adaptar y aprovechar los recursos disponibles y de bajos niveles de externalización. Este proceso es más conocido en la literatura como la producción de Novelties y es característico en la agricultura familiar ${ }^{6}$ (Van der Ploeg, 2010).

La producción estuvo marcada desde sus inicios por una importante variabilidad, tanto en lo relativo a los volúmenes de producción como al tiempo transcurrido para la incorporación de una nueva camada de pollitos bebé (Figura 1).

Esto sucede como consecuencia del proceso de ir ajustando un esquema productivo, con todas las complicaciones que ello trae aparejado, y paralelamente tener que ir abriendo nuevos canales comerciales. No solo eso, sino que una vez abiertos debe garantizarles una continuidad en términos de disponibilidad y calidad del producto.

Es este juego entre oferta y demanda una de las cuestiones más complejas de equilibrar en las prime-

\footnotetext{
Ejemplos de este proceso lo constituyen la incorporación de un nuevo corral, construido por el mismo productor adaptando una vieja caja de su camioneta, la fabricación de bebedero con caños de PVC calados, adaptando en sus extremos sifones de soda para vaciarlos y limpiarlos, el corral para "sacar a pasear" los pollos, entre otros.
} 
Cuadro 1. Producción de pollos anual de Don Ricardo (10/2011 al 07/2014).

Table 1. Don Ricardo's annual chicken production $(10 / 2011$ al 07/2014).

\begin{tabular}{ccccc}
\hline & \multicolumn{4}{c}{ Período } \\
\cline { 2 - 5 } & $\mathbf{2 0 1 1}$ & $\mathbf{2 0 1 2}$ & $\mathbf{2 0 1 3}$ & $\mathbf{2 0 1 4}$ \\
\hline $\begin{array}{c}\text { Cantidad de pollos } \\
\text { comprados }\end{array}$ & 150 & 850 & 800 & 470 \\
\hline
\end{tabular}

ras etapas de la vida de un emprendimiento (Porter, 1996), y en el caso de Don Ricardo se puede observar claramente dicha complejidad: en el año 2012, gracias a las inversiones que había realizado el año anterior, el productor logró incrementar sus volúmenes de producción, pero se enfrentó con algunos problemas comerciales que lo obligaron a retraerse temporalmente en sus volúmenes ${ }^{7}$. El año 2013 en cambio estuvo marcado por una demanda más estable de pollos, pero el productor sufrió importantes problemas de sanidad por un parásito en los corrales, y fue recién a mitad de año cuando pudo identificar la solución al problema, pero ya había perdido el $90 \%$ de su producción. En el año 2014 se encontró nuevamente con algunos problemas para colocar la producción, al punto que llegó a no faenar animales por falta de lugar en el freezer para almacenarlos, y tuvo que retrasar la incorporación de una nueva tanda hasta solucionar este inconveniente.

Situaciones similares a las descritas sin duda tendrían consecuencias muy importantes en empresas de corte capitalista, pero en el caso estudiado tanto la di-

En las primeras tandas, tuvo algunos inconvenientes para encontrar el canal adecuado donde vender su producción, luego recibió algunos reclamos por parte de sus clientes por el dispar tamaño de los pollos y la cantidad de grasa que tenían, con lo cual se vio en la necesidad de ajustar la composición de las raciones. versificación productiva como las estrategias de no mercantilización y su autonomía relativa del mercado, son elementos que le permitieron soportar este proceso de adaptación y aprendizaje sin sobresaltos, y mantenerse en una tendencia productiva y comercial creciente.

\section{Caso 2: El sistema de producción de Don Francisco.}

Don Francisco se inició en la actividad lechera caprina en el año 1997. La explotación está ubicada en el paraje denominado El Polear a unos $5 \mathrm{~km}$ del centro de la ciudad de La Banda y a $12 \mathrm{~km}$ de la ciudad capital. El predio se compone de 5 hectáreas propias y 5 hectáreas cedidas por un vecino; aprovecha además 30 hectáreas lindantes, donde hace pastorear sus animales. Dentro de las 5 hectáreas tiene dos hectáreas con verdeos de verano (maíz y sorgo), una hectárea con avena (verdeo de invierno) y otras dos hectáreas de alfalfa para pastoreo. Mayores detalles respecto a la dimensión técnicoproductiva como económica del predio se encuentran en Paz et al. (2011).

La estructura familiar está compuesta por Don Francisco (de 60 años) y su esposa Ángela (64), quien actualmente está jubilada como maestra de escuela. El matrimonio tiene dos hijas, pero ellas no residen en el predio ya desde hace mucho tiempo, y consecuentemente nunca integraron el esquema productivo de la explotación (al menos desde su reconversión productiva a fábrica de quesos de cabra). La ausencia de las hijas en el predio abre una línea de debate sobre la continuidad o sostenibilidad de la explotación familiar; sin embargo, este aspecto no será abordado en este trabajo.

Don Francisco también interviene directamente tanto en el proceso productivo como en el de gestión de la explotación. Doña Ángela lleva el manejo de la casa y suele encargarse de la atención al público. Además de ellos dos, se contrata una persona de manera permanente para llevar adelante las tareas de la producción

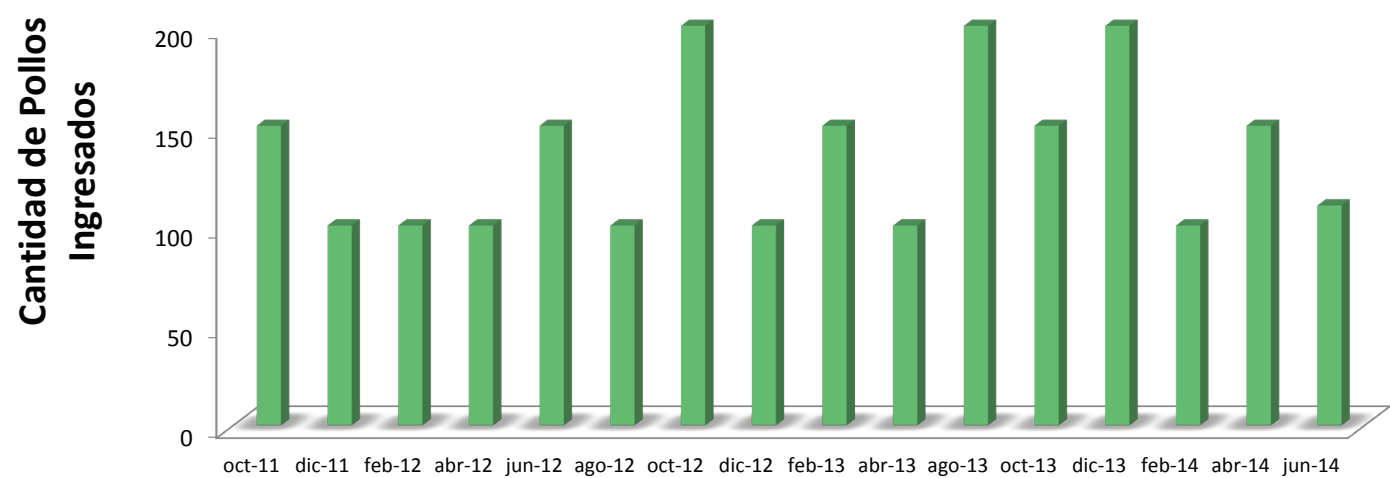

Meses de Ingreso de Pollos

Figura 1. Cantidad de pollitos ingresados en los corrales para engorde (2011-2014).

Figure 1. Number of admitted chicks for fattening (2011-2014). 
pecuaria en general. También se contratan a dos mujeres para realizar trabajos de higiene, desinfección, limpieza, ordeño del tambo y apoyo en la elaboración de los principales productos (quesos y chacinados). Sus servicios son prestados anualmente, aunque se diferencian en contratos laborales según las distintas estaciones.

El sistema pecuario es diversificado y está compuesto por 2 equinos, 2 vacas y 2 terneras, 109 cabras, 3 reproductores caprinos; 7 cerdas reproductoras. Los cabritos, capones y lechones varían de acuerdo al proceso productivo. Además tiene aves de corral (22 gallinas ponedoras, un gallo y 6 gansos) y unas cuantas colmenas ubicadas en el monte. Parte de la producción se orienta al autoconsumo y otra importante, a la venta. La producción primaria está fuertemente integrada al proceso agroindustrial, buscando una mayor vinculación de los recursos tendientes a generar mayor valor agregado.

El proceso agroindustrial se desarrolla a partir de la conversión de leche a queso y de producción de carne (especialmente la proveniente de los cerdos) a chacinados o embutidos; es allí donde se busca un agregado de valor de la producción primaria, en especial la leche y la carne de cerdo.

La producción de chacinados se lleva a cabo en otoño-invierno. Los porcinos que se sacrifican son criados con insumos provenientes de la propia explotación (suero, forrajes, granos, entre otros) y de compra externa como soja o maíz. Los productos fabricados son salame, chorizo, morcilla y arrollado de cerdo, y se orientan fundamentalmente al mercado. Dichos productos se fabrican en una sala de producción bien acondicionada, donde también se hacen los quesos, cuidando siempre de las buenas prácticas de higiene y sanidad. Hay tres heladeras para mantener la cadena de frío.

La producción de quesos es la actividad principal desempeñada en la explotación y se realiza diariamente durante todo el año de forma artesanal. Los quesos tienen como principal insumo la leche que proviene de la misma explotación. El ordeño es manual y comienza en la mañana bien temprano. El proceso de elaboración del queso termina aproximadamente al mediodía.

Paralelamente, durante la época de clases, el establecimiento recibe la visita de escolares que es un servicio rentado que puede ser definido desde el agroturismo. Doña Ángela coordina la visita y realiza la recepción de los niños y sus maestros pues cuenta con experiencia como docente. Los niños que asisten a la visita de la explotación permanecen en ella tres horas aproximadamente. Durante el paseo participan en la alimentación de los distintos animales, recogen los huevos, juegan con los cabritos, observan a los vacunos y los equinos, ven cómo se ordeñan las cabras. Luego se les muestra cómo elaboran los quesos, y la visita termina con un desayuno con pan, miel y leche. Al finalizar la visita los maestros aprovechan la posibilidad de com- prar productos elaborados artesanalmente. Es esta una forma de dar a conocer los productos y atraer al cliente, ya que mientras los escolares desayunan, se ofrece una degustación de los productos a los adultos. En el último año recibió más de 700 alumnos.

\section{Los circuitos cortos en la comercialización}

Históricamente, las dos principales empresas productoras de queso de cabra de la provincia (FUNDAPAZ y La Carola S.R.L.) ubicadas en las cercanías de la explotación de Don Francisco, han intentado colocar su producción fundamentalmente en los comercios ubicados en las ciudades de La Banda y Santiago del Estero. En estos mercados, las empresas lograron colocar en promedio alrededor de $1.200 \mathrm{~kg}$ de queso de cabra al año, debido a que se han topado con serias dificultades para ampliar el mercado. Sin embargo, Don Francisco con su pequeño emprendimiento ha logrado colocar en el año 2013 más de $2.000 \mathrm{~kg}$ de queso de cabra en estos mismos mercados. Su estrategia de venta está puesta en la utilización y comprensión del funcionamiento de los circuitos cortos y descentralizados que vinculan directamente (cara a cara) la producción y el consumo. Los escolares y las personas que visitan la explotación, los clientes permanentes a los cuales les lleva el queso a su domicilio, la venta a un local de delicatessen de renombre en la ciudad capital y un comprador extralocal que distribuye el queso en distintos puntos del país son los circuitos más importantes.

El proceso de construcción de estos circuitos fue lento, pero orientado estratégicamente a un objetivo en el largo plazo: sus primeros pasos en la actividad los dio como tambero caprino, y durante un tiempo solo entregó leche a las usinas lácteas locales, lo que le permitió un conocimiento en la producción primaria de leche de cabra al tiempo que le permitió una estabilidad financiera. Luego asistió a una capacitación donde aprendió a hacer quesos y comenzó a producirlos a baja escala, en su propia casa y con su propia leche. Así, paulatinamente comenzó a descubrir circuitos de comercialización alternativos a los utilizados por las fábricas, vendiendo los quesos en forma directa entre allegados, vecinos y clientes antiguos (que ya le compraban embutidos), mediante el sistema de entrega a domicilio o venta en su propia explotación. Paralelamente, y a medida que iba perfeccionando la técnica de elaboración y abriendo nuevos canales de comercialización, el productor fue reduciendo la entrega de leche a las fábricas, hasta llegar en el año 2007 a una demanda de quesos acorde similar a su capacidad de producción de leche y a partir de ese momento se produjo una reorientación definitiva de su plan de negocios hacia la producción de quesos.

Este proceso deja en evidencia la capacidad de la agricultura familiar para ir fortaleciéndose en forma 
paulatina, paso a paso y sin que el tiempo apremie, ya que al contrario de lo que sucede con las explotaciones capitalistas, lo que se busca no es un retorno sobre la inversión, sino obtener una valorización cada vez mayor de la mano de obra familiar a través del agregado de valor en la producción (Van der Ploeg, 2010).

De esta forma para Don Francisco, un queso de calidad, el uso de buenas prácticas tanto en la producción de leche como en la elaboración del queso, un precio final que está por debajo de la competencia ${ }^{8}$ y circuitos de cadena corta constituyeron la clave de su estrategia de comercial.

El uso de estos circuitos comerciales, distintos de los de cadena larga y menos formalizados, difícilmente responda a los formatos tradicionales de comercialización. Sus propias características los hacen difícilmente identificables y, en consecuencia, resulta complejo ponderarlos monetariamente. En síntesis, se podría decir que existe una economía invisible pero no por ello carente de valor comercial.

Desde la perspectiva de un economista clásico, la venta de quesos por parte de Don Francisco puede ser definida como un mercado tradicional en cuanto hace referencia a canales de distribución informales, productos poco diferenciados, variaciones relativamente importantes en calidad y homogeneidad, poca transparencia en el precio y, fundamentalmente, posibilidades de realizar las transacciones en el momento que el productor lo necesite (Durstewitz y Escobar, 2006). Sin embargo, ello no quita que también presente algunas características de un mercado dinámico en cuanto puede absorber una importante cantidad de los bienes producidos en la finca, como también en el propio territorio (Schejtman y Ramírez, 2004). En una síntesis de varias experiencias en una convocatoria realizada por Chorlavi, se llega a concluir que

[...] "la connotación de mercados dinámicos no sólo se observa en espacios de venta extralocales formales. Por el contrario, se ha observado que mercados locales con altos grados de informalidad también pueden resultar en espacios de mercadeo dinámico para los territorios pobres y marginados " [...] (Ramírez et al., 2007: pág. 8).

Sin embargo, la construcción de estos circuitos cortos y no formales logra su competitividad a partir de escalas mínimas de producción y bajos puntos de equilibrio, a través de fuertes procesos de artesanalidad de la producción y de intensificación (Paz et al., 2006).

Precisamente, una baja inversión externa, ausencia de categorías económicas como las amortizaciones, salarios, pagos de intereses por créditos o pagos por impuestos y un despliegue de estrategias de no mercantilización, en un marco de mayor endogeneidad y sustentabilidad de los sistemas de producción permite obtener una producción a bajo costo y por lo tanto ser más competitiva.

\section{Ajustando paso a paso los procesos productivos con la demanda del mercado.}

Los volúmenes de producción de los quesos están fuertemente asociados a la capacidad de gestión que tenga la explotación para generar un mercado para su colocación. Debe haber una cierta sincronización entre la oferta y la demanda, a los efectos de no generar un excedente que luego tendrá que mal vender o consumir. Don Francisco en estos últimos 5 años ha venido abriendo mercados siempre desde los circuitos cortos, privilegiando las relaciones interpersonales y espacios comerciales donde él puede de alguna manera intervenir como formador de precios.

Aunque no existe un plan de mercado formalmente establecido; si lo que hay, es un conocimiento profundo sobre costos de producción, calidad del producto, tipos de clientes, tendencia y condiciones del mercado, entre otros.

Aquí hay una preocupación que es general a todas las actividades productivas y en especial a la pequeña producción artesanal y es cómo lograr ser competitivo en un mercado donde la falta de competitividad se la castiga rápidamente, teniendo que retirarse la empresa del mercado precisamente por no cumplir con esta condición (Paz et al., 2006). Don Francisco conoce de las limitaciones y las ineficiencias que están presentes en su predio. Las mismas actuaron constantemente desde el inicio mismo de la producción y rápidamente se plantea la necesidad de articular de manera eficiente entre la producción de leche y la elaboración del queso además de sincronizar con un mercado que tiene que ser dinámico a tal producción. Poner en régimen su proceso productivo primario e industrial sin tener que soportar pérdidas constituye uno de los principales desafíos al momento de emprender cualquier negocio (Farrel y Thirion, 1999).

El Cuadro 2 muestra algunos parámetros técnicoproductivos que tomados aisladamente posiblemente resulten simples datos de la explotación, pero, considerados en conjunto, expresan estrategias productivas que se relacionan con las estrategias de mercadeo.

Como se comentó, Don Francisco va ganando espacios comerciales, es decir va construyendo mercados dinámicos para su propia producción. Esto va traccionando la producción de quesos que va en aumento pero a la vez, en el propio proceso de producción de leche va ajustando su sistema productivo en términos de mejorar la alimentación, la genética y la sanidad tendientes a aumentar los parámetros técnico-productivos de la leche en especial el tenor graso, proteico y la calidad bacteriológica de la leche. La mejora de tales parámetros repercute en el rendimiento quesero, logrando una mayor eficiencia de reconversión de leche a queso en el proceso agroindustrial. Finalmente, la estacionalidad de la producción de leche va también mejorando, obte- 
Cuadro 2. Parámetros técnico-productivos de la producción de leche y queso de Don Francisco.

Table 2. Technical and productive parameters of Don Francisco's milk and cheese.

\begin{tabular}{lrrrrr}
\hline \multicolumn{5}{c}{ Producción anual de leche de cabra (Litros) } \\
\hline & 2009 & 2010 & 2011 & 2012 & 2013 \\
Litros/año & 12.297 & 13.534 & 16.867 & 19.850 & 21.185 \\
Rto. Quesero & 8,18 & 8,02 & $\mathrm{~s} / \mathrm{d}$ & $\mathrm{s} / \mathrm{d}$ & 7,50 \\
Kg de queso & 1.503 & 1.688 & 2.103 & 2.475 & 2.825 \\
Verano/Invierno & 3,02 & 3,45 & 3,06 & 2,72 & 2,05 \\
\hline
\end{tabular}

niendo un litro de leche de invierno por cada dos litros de leche de verano. Como mención y para entender esto último, la mayor demanda de quesos suele ser en invierno donde hay menos leche y donde los precios son más altos.

Este simple ejemplo muestra la complejidad de poner a punto de manera eficiente los procesos productivos, e ir buscando los ajustes necesarios para al fin de cuentas bajar los costos de producción y obtener un queso de mejor calidad, que en definitiva se traduce en mayores ganancias. Esto es lo que damos por llamar la producción paso a paso, que resulta una característica muy propia de la agricultura familiar.

\section{Caso 3: El sistema de producción de Colonia Jaime}

Colonia Jaime es una comunidad agropecuaria fundada en el año 1932. La explotación está ubicada en la Ruta Nacional 34, Km 711, departamento Robles-Santiago del Estero, sobre un predio de 607 hectáreas de las cuales 365 están ocupadas actualmente con actividades agropecuarias y la casa comunal.

La comunidad está conformada por aproximadamente 80 personas que se distribuyen en 25 familias. Las mujeres se dedican exclusivamente a las tareas domésticas y la elaboración de productos elaborados (quesos, pan casero, etc.). Además son quienes reciben las visitas de turistas y escuelas de la provincia que llegan a conocer el particular sistema de vida de la colonia. Por su parte, los hombres se encargan de las diferentes actividades agropecuarias que se llevan adelante en la explotación, mediante la constitución de grupos de trabajo de entre tres y cuatro personas.

El sistema pecuario es diversificado, y en él se destaca la actividad ganadera con aproximadamente 400 cabezas, de las cuales alrededor de 100 se mantienen rotativamente en ordeñe. La producción de leche asciende a 1.200 litros diarios en promedio y es obtenida en dos turnos: por la mañana 700 litros y por la tarde 500 litros. De esta producción, el 84\% es entregado a Lactiband (una empresa de lácteos que funciona en la ciudad de La Banda), el resto es destinado al autocon- sumo y el excedente es vendido en las "granjas comerciales" de la colonia9.

Para la actividad porcina se cuenta con 47 madres y 3 reproductores, obteniendo un promedio de 8 lechones por parto. Los lechones obtenidos son destinados tanto al autoconsumo como a la venta, pero no se utilizan para la fabricación de embutidos ni fiambres. Aproximadamente se venden 12 lechones a la semana, pero este volumen se incrementa a 300 mensuales en diciembre por la gran demanda que existe en las fiestas de fin de año.

En cuanto a la actividad avícola, Colonia Jaime recibe aproximadamente 900 pollitos híbridos por semana, que se engordan y son faenados para venderse posteriormente como pollos parrilleros. Además disponen de unas 80 colmenas.

Como sucede con el caso anteriormente descrito, la producción primaria está fuertemente integrada al proceso agroindustrial, buscando una mayor vinculación entre los distintos recursos con el objetivo de generar mayor valor agregado. Esto se logra a partir de la conversión de leche a queso (sólo para autoconsumo), la producción de miel y la producción de carne: la proveniente de capones a chacinados o embutidos (chorizos, morcillas, arrollados) y de pollos híbridos a pollos de granja. Para ello cuentan con instalaciones muy equipadas, entre las cuales se destaca un tambo con ordeñadora mecánica de ocho bajadas, una sala de extracción de miel con máquinas extractoras y purificadoras, una fábrica de chacinados con aberturas y cámara frigorífica de doble propósito. Además, después de dos años de pruebas y estudios, recientemente se puso en funcionamiento un Biodigestor que opera a partir del gas que se obtiene del guano de las vacas de la explotación. La aplicación de abonos disueltos mediante riego por goteo o "fertirriego" constituye también otra novedad tecnológica que ha sido incorporada para el desarrollo de la actividad fruti hortícola (en una extensión de 8 hectáreas).

\footnotetext{
Locales comerciales que tienen alquilados en la ciudad de La Banda, administrados y atendidos por los mismos habitantes de la colonia, donde se comercializa una parte de la producción generada en la misma explotación, como así también otros productos que no se producen en la colonia y son obtenidos de proveedores.
} 
Colonia Jaime también cuenta con 2 locales de venta propios en la ciudad de La Banda, donde centraliza la venta de los productos generados por las actividades agropecuarias y complementarias. Al respecto, los comuneros destacan que "muchas amistades se iniciaron en los interiores de estos dos locales y sin lugar a dudas, el mayor beneficio es el intercambio de principios, culturas, experiencias y trabajo".

El sistema de producción de Colonia Jaime representa así un caso particular de agricultura familiar, ya que si bien se basa en un particular esquema comunal de producción, presenta estrategias económico-productivas de bajos niveles de externalización, coproducción hombre-naturaleza ${ }^{10}$, productos diferenciados, circuitos cortos de comercialización y recursos vinculantes a partir de procesos basados en la intensificación de la mano de obra de las familias que integran la comunidad. Este estilo de agricultura se denomina agricultura económica (Van der Ploeg, 2006)

\section{Estrategias de producción y agregado de valor}

La producción de chacinados ha sido históricamente una de las principales actividades de Colonia Jaime. Desde su fundación, los primeros habitantes (descendientes de inmigrantes italianos, yugoslavos, españoles y algunas familias santiagueñas), fabricaron los primeros chorizos italianos y criollos a partir de recetas tradicionales de sus familias. La elaboración de los chacinados era realizada de forma artesanal en épocas de invierno y la producción estaba destinada solo al consumo interno. En esa época, la carencia de instalaciones para su conservación obligaba a los comuneros a desplegar ciertas estrategias para disponer de chacinados por dos o tres meses (dependiendo del frio invernal). Los lugares de almacenamiento eran sótanos o piezas cerradas, aunque en ocasiones se llegó a conservarlos en baldes totalmente cubiertos con grasa.

Los capones eran criados a campo y alimentados en base a maíz y restos de alimentos provenientes de la propia explotación. Durante muchos años se mantuvo esta modalidad de crianza de los cerdos y de elaboración de los chacinados, hasta que el sistema entró en una profunda crisis económica que puso en duda su sostenibili$\mathrm{dad}^{11}$. Los comuneros no contaban con antecedentes en

10 En su sentido más general, la agricultura puede ser entendida como una coproducción: el encuentro entre lo social y lo natural (Toledo, 1990). En ese marco, la agricultura puede ser vista como la continua interacción y transformación mutua entre las personas y el medio natural que las circunda. La humanidad utiliza la naturaleza, y por medio de esta práctica la transforma. Pero a su vez el hecho de utilizar la naturaleza, también pone su impronta en la propia sociedad. De esta forma, la coproducción configura y reconfigura lo social del mismo modo que lo hace con lo natural (Van der Ploeg, 2013).

11 Esta crisis se generó debido al incremento de la cantidad de comuneros y el consecuente aumento de la cantidad de insumos mercantiles que eran necesarios para satisfacer las necesidades de autoconsumo de la comunidad (elementos de limpieza, insumos utilizados en las distintas producciones, tela para ropa, etc.). ventas, marketing y organización de empresas, pero en los debates internos se observaba que la única salida a esta situación era la comercialización organizada de sus productos. Esto significaba incrementar los volúmenes de producción, rediseñar estrategias de organización de la mano de obra para una mejor crianza y elaboración de la producción, así como construir mercados que se adecúen no solo a sus niveles de producción sino también a los objetivos que como comunidad perseguían, asegurando la sostenibilidad del modelo.

La estrategia desarrollada estuvo basada en producir la mayor cantidad posible de insumos dentro de la misma finca (para mantener bajos costos de producción), quedando en evidencia el fuerte grado de endogeneidad y control sobre sus recursos, como alternativa a la externalización y vulnerabilidad que generaría una producción basada en la compra de insumos externos en el mercado (Paz y Bruno, 2013).

\section{Construyendo mercados para los chacinados}

El punto de partida para que Colonia Jaime comience a llegar con sus productos al mercado fue una invitación del intendente de la ciudad de La Banda a participar como expositores en la feria organizada para aniversario de la ciudad (año 1992). En esa feria la comunidad participó ofreciendo embutidos, chacinados, panificados, frutas y quesos, todos ellos elaborados en forma artesanal y a partir de prácticas tradicionales de producción. Este esquema, obtenido a partir de la intensificación de la mano de obra familiar, habla de un saber hacer que no solo vincula al hombre con la naturaleza en un proceso de coproducción y coevolución ${ }^{12}$, sino también de una continuidad entre el presente, el pasado y el futuro (Van der Ploeg, 2010).

El puesto de Colonia Jaime, atendido durante cinco días por tres comuneros, resulto ser un éxito. Es así como comenzaron a participar en ferias organizadas en distintas localidades de la provincia. Estas experiencias como feriantes, sumadas a las obtenidas por "la venta a pedido"13 (como le llamaban ellos) fueron claves para comprender el funcionamiento de los circuitos cortos de comercialización, aprovechando los beneficios en mate-

12 De acuerdo a Norgaard (1994) el concepto explica el desarrollo paralelo a lo largo de la historia de la naturaleza y la sociedad, de forma tal de que cada una de ellas depende de la otra para continuar su reproducción. La teoría coevolucionista pone en relieve que: 1) los sistemas agrícolas se deben considerar como un sistema integral y parte de sistemas mayores, 2) estos sistemas no son estáticos, 3) las personas y su forma de pensar se encuentran dentro del proceso, 4) la legitimidad del proceso cultural y experimental de los agricultores.

13 Se trata de una forma particular de venta a domicilio: una persona levantaba pedidos durante los primeros cuatro días de la semana en la ciudad; los miércoles se carneaban capones, los jueves se dejaba enfriar la carne, los viernes por la mañana se preparaba el chorizo y por la tarde ya se tenían listas las bolsitas con los pedidos de cada cliente, las cuales eran distribuidas los sábados por la mañana. Los primeros clientes que utilizaron este sistema fueron amigos, familiares y conocidos de los comuneros. 
ria de valor agregado y autonomía productiva que les proporcionaba el "cara a cara" entre productor y consumidor.

En el año 1993 un familiar le ofrece a Colonia Jaime un pequeño local comercial en la ciudad de La Banda para vender su producción, a cambio de que la colonia se haga cargo de soportar los impuestos del mismo. Contar con este espacio físico fijo donde colocar sus productos en la ciudad, significó un importante salto para la comunidad, tanto cualitativamente como cuantitativamente. En primer lugar, este hecho les permitió acortar la distancia entre el campo y la ciudad mediante un sistema de venta directa en una tienda propia: una alternativa comercial no muy frecuentemente observada en los estudios sobre circuitos cortos (CEPAL, $2013 b$ ). En segundo lugar, este esquema le permitió a Colonia Jaime capturar un mayor valor por su producción, ahorrar en transporte y crear valor a partir de activos inmateriales tales como una identidad en el producto, anclaje territorial, autenticidad y lazo social.

En la actualidad la comunidad ha profundizado esta estrategia, y ya cuenta con dos comercios en la ciudad de La Banda ${ }^{14}$ mediante los cuales comercializan su producción de chacinados. Ambos son atendidos solamente por miembros de la colonia.

Al igual que en el resto de los casos estudiados, resulta evidente la importancia de las relaciones sociales no mercantilizadas (Smith, 1984) no solo por la forma de ir abriendo y construyendo el mercado, sino también en la conformación de una "alianza" entre productores y consumidores que manejan pautas productivas y comerciales comunes, donde se valora la producción artesanal, saludables y de temporada, y se representa una opción más equitativa, solidaria y responsable, que respeta y no compromete la autonomía de la producción familiar.

14 La segunda boca de expendio surgió como consecuencia del fallecimiento de otro familiar de la colonia, quien les dejó una casa ubicada en una de las principales avenidas comerciales de la ciudad de la Banda.

\section{El paso a paso en el proceso productivo y su relación con la demanda del mercado}

En los 20 años que Colonia Jaime lleva comercializando sus productos, los comuneros han estado siempre preocupados por asegurar un equilibrio entre la oferta y la demanda, para no generar riesgos en cuanto a excedentes de producción. Destacan que si bien no pudieron consolidar (hasta ahora) una gestión de todo el sistema de producción que permita alcanzar un control total a modo de un plan de negocio o de mercado, el conocimiento que se viene obteniendo, a partir de los trabajos con la naturaleza y los vínculos con los clientes y proveedores de algunos productos, constituye el recurso de mayor importancia que conecta todos los eslabones de una larga cadena de producción, circulación y consumo.

$\mathrm{Al}$ respecto, Milone (2013) argumenta que en la actualidad, los agricultores buscan reinventar los mercados para sus productos a partir de la defensa y valor de todos sus activos: la fuerza de trabajo familiar, la propiedad intelectual, el conocimiento y la tierra. Entonces a partir de esto buscan crear nuevos mercados, reinventar sus prácticas agrícolas y encontrar nuevos usos para los recursos disponibles en las fincas.

En el caso de Colonia Jaime se observa el paso de la producción para el autoconsumo a una producción para el mercado. Los ajustes se dieron tanto al nivel de la mano de obra familiar involucrada en la actividad, como al nivel de la producción (Figura 2) e incorporación de tecnología (con maquinarias e importantes inversiones en infraestructura ${ }^{15}$ ).

Sin embargo, y a pesar de esta fuerte transformación hacia una producción mercantil, la explotación aún conserva su propia autonomía, brindada sobre todo por

\footnotetext{
Desde hace dos años se puso en funcionamiento una sala de elaboración de chacinados, que cuenta con cámaras frigoríficas y maquinarias de gran capacidad de producción (tales como picadora, amasadora y embutidora).
}

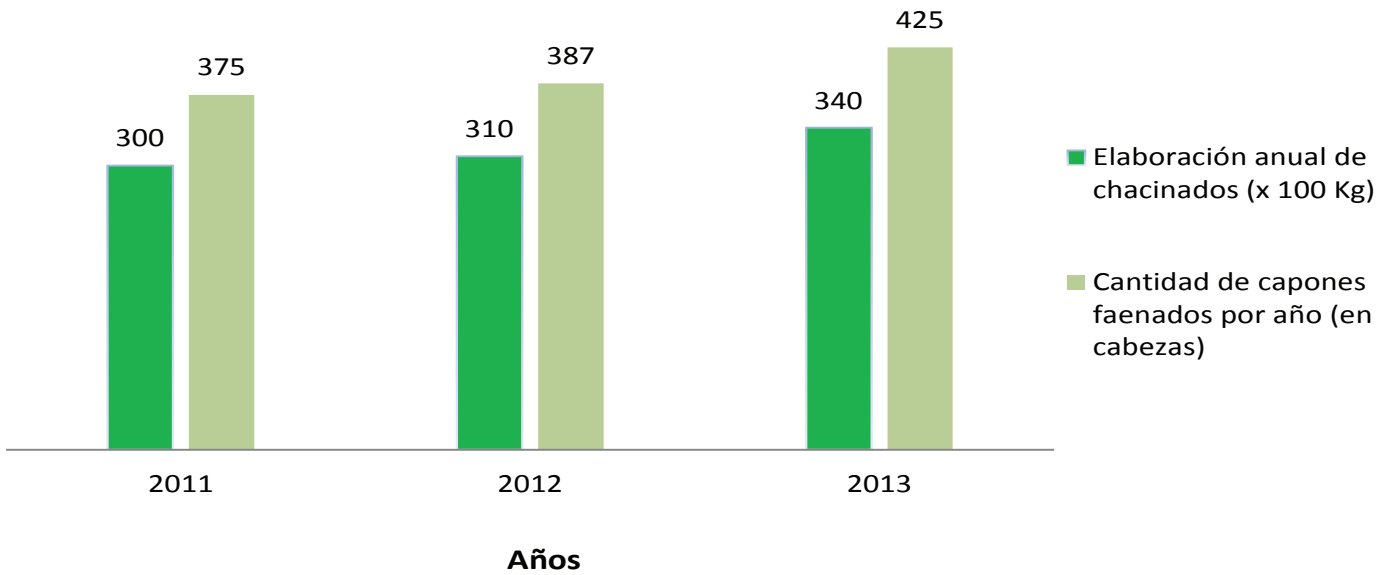

Figura 2. Producción de chacinados de cerdo y capacidad de faena en Colonia Jaime (2011-2013).

Figure 2. Hog cold cuts production and slaughter capacity in Colonia Jaime (2011-2013). 
las características de su mano de obra (provista por los comuneros), la utilización de insumos no mercantilizados en su proceso productivo (el Biodigestor es un claro ejemplo), bajos niveles de externalización y, sobre todo, su particular forma de relacionarse con el mercado.

\section{CONCLUSIONES}

Los casos presentados constituyen experiencias particulares de agricultura familiar no extrapolables a otros espacios rurales, sin embargo del estudio de estas explotaciones se pueden extraer distintas reflexiones que sin duda resultan útiles al momento de pensar en políticas públicas orientadas a potenciar el sector.

En relación al actor social se demuestra que lejos de ser sujetos pasivos que solo resisten y se adaptan a un medio capitalista que lo agrede y busca subsumirlo, van generando respuestas activas a la creciente mercantilización, permitiendo que el mercado y otras fuerzas "externas" ingresen al mundo de la explotación familiar, e integrándolos dentro de sus propias estrategias agrícolas, pero al mismo tiempo cuidando de mantener un grado relativo de autonomía en materia de toma de decisiones.

Pero no hay que perder de vista que una parte importante de este sector se ubica en una situación de dependencia, marginación y privación, y justamente es ahí donde este actor social despliega su capacidad de actuar y, en ese contexto, es clave el modo que tiene para relacionarse con el mercado (Long, 1986). El objetivo es lograr un esquema comercial que le permita una máxima flexibilidad, movilidad y libertad, creando un espacio de maniobra que les facilite su contracción y expansión en el momento considerado oportuno (Paz, 2008).

Los casos expuestos demuestran que lograr esta "autonomía relativa" es posible, ya que mediante decisiones comerciales concretas, los productores continúan controlando la organización de su propio trabajo. De esta forma, se podría sostener que la actual fortaleza y viabilidad de la producción familiar no descansa en su grado de orientación al mercado, sino en la forma en la que el productor logra ir encontrando los circuitos comerciales apropiados para no comprometer la autonomía en su producción.

En relación a las características de los circuitos comerciales se observa que la agricultura familiar tiende a generarlos mediante un proceso de construcción paulatino, echando mano para ello a las redes de relacionamiento social en las que se halla inmersa, y la forma de relacionarse que genera este tipo de intercambio, por lo general termina derivando en esquemas comerciales de circuitos cortos o de proximidad.

Este proceso, a su vez, se encuentra articulado con -y condicionado por- las propias características de la producción, que como se observó en los estudios de caso, se basa en volúmenes de producción que resultan manejables, flexibles y, en determinadas situaciones, sufre importantes variaciones de calidad como consecuencia de "la puesta a punto" de un proceso productivo artesanal. En ese contexto, la agricultura familiar difícilmente pueda admitir la imposición de condiciones en materia de fijación de precios, reglas impersonales de higiene, calidad, estandarización u homologación como las que utilizan comúnmente los grandes circuitos comerciales.

Con esto no queremos decir que no se controle la higiene, la calidad o se exijan determinadas homologaciones en los productos provenientes de la agricultura familiar, sino que es necesario evaluar la creación de reglas flexibles, que contemplen las particularidades de este sector.

Un sistema de producción que obtiene sus ventajas competitivas a través de la intensificación de la mano de obra familiar, diversificación de su producción, bajos niveles de externalización, endogeneidad, control sobre los recursos y el despliegue estrategias de no mercantilización (Van der Ploeg, 2010), requiere de canales de venta mucho más flexibles, informales, rápidos y directos como en estos casos han demostrado serlo las distintas alternativas comerciales utilizadas por los productores.

Finalmente, no se puede dejar de mencionar el rol que debería cumplir el estado como diseñador de políticas públicas, captando estas especificidades y actuando como facilitador para que los alimentos de la agricultura familiar lleguen a la población. En ese marco de estas experiencias se deduce que debería existir un fuerte apoyo en los primeros momentos de la apertura comercial de la explotación, que genere nuevas alternativas de venta, amplíe las posibilidades del productor de generar vínculos comerciales, pero que a su vez garantice su estabilidad financiera y no comprometa su autonomía. Ejemplos de estas acciones serían la construcción de centros de acopio y venta comunitarios, mercados concentradores, puntos de venta en las ciudades de productos provenientes de la agricultura familiar, o hasta programas de compra estatal de utilicen sus productos (como por ejemplo el Programa de Adquisición de Alimentos de Brasil).

En suma, en este trabajo se pretende demostrar que potenciar la agricultura familiar es posible, pero para ello antes resulta necesario comprender y respetar sus particularidades.

\section{REFERENCIAS}

Andrade de Almeida Cunha, A. R., 2013. Las centrales mayoristas de abasto y los circuitos cortos en América Latina, in: Agricultura familiar y circuitos cortos. Nuevos esquemas de producción, comercialización y nutrición. CEPAL. Serie de Seminarios y Conferencias № 77, pp. 69-74. 
Aubron, C., Hernández, M., Lacroix, P., Mafla, H., Proaño, V., 2013. Producción campesina lechera en los países andinos. Dinámicas de articulación a los mercados. Sistema de Investigación sobre la Problemática Agraria en el Ecuador (SIPAE). Quito.

Barbosa-Calvacanti, J. S., 2004. New Challenges for the Players in Global Agriculture and Food. International Journal of Sociology of Agriculture and Food 12, 29-36.

Berdegué, J., Nazif, I., 1988. Sistemas de Producción Campesinos. Serie Libros GIA № 4. Santiago.

Cáceres, D., Silvetti, F., Soto, G., Rebolledo, W., 1997. La adopción tecnológica en sistemas agropecuarios de pequeños productores. Agro Sur 25(2), 123-135.

CEPAL 2013a., Perspectivas de la agricultura y del desarrollo rural en las Américas: una mirada hacia América Latina y el Caribe. Serie de Seminarios y Conferencias, Santiago de Chile.

CEPAL 2013b., Agricultura familiar y circuitos cortos. Nuevos esquemas de producción, comercialización y nutrición. Serie de Seminarios y Conferencias. Santiago de Chile.

Chauveau, C., Taipe, D., 2012. Circuitos alternativos de comercialización. Estrategias de la agricultura familiar campesina. Inventario, impacto, propuesta. Editado por el Ministerio de Agricultura, Ganadería, Acuacultura y Pesca de Ecuador, Quito.

Chiodo, L., 2010. Hipermercados en América Latina: Historia del comercio de alimentos, de los autoservicios hasta el imperio de cinco cadenas multinacionales. Antropofagía, Buenos Aires.

Durstewitz, P., Escobar, G., 2006. La vinculación de los pequeños productores en los mercados. Documento RIMISP. Serie Intercambios (63). http://www.rimisp.org/boletin_intercambios/index_boletin.php?id_boletin=60 (acceso, 29.06.2014).

FAECyS., 2012. Relevamiento sobre supermercados en Argentina. Informe. http://www.faecys.org.ar/Informe_ Supermercados.pdf (acceso, 25.08.2014).

Farrel, G., Thirion, S., 1999. La competitividad territorial. Observatorio Europeo Leader.

Hebinck, P., Schneider, S., Van der Ploeg, J. D., 2015. The construction of new, nested markets and the role of rural development policies. Some introductory notes, in: Hebinck, P., Van der Ploeg, J.D., Schneider, S. (Eds.), Rural Development and the Construction of New Markets. Routledge, pp. 1-15.

INTA (Instituto Nacional de Tecnología Agropecuaria)., 2010. Las ferias de la Agricultura Familiar en la Argentina. Comercialización y Financiamiento. Ediciones INTA, Buenos Aires, Argentina.

Jacoby, E., 2013. Circuitos cortos, salud y nutrición en América Latina, in: Agricultura familiar y circuitos cortos. Nuevos esquemas de producción, comercialización y nutrición. CEPAL. Serie de Seminarios y Conferencias № 77, pp. 27-31.

Lacroix, P., Cheng, G., 2014. Ferias y mercados de productores: hacia nuevas relaciones campo-ciudad. VSF-CICDA - CEPES, Quito.

Long, N., 1986. Commoditization: thesis and antithesis, in: Long, N., Van der Ploeg, J., Curtin, C., Box, L. (Eds.), The commoditization debate: labour process, strategy and social network. Departments of Sociology (17). Agricul- tural University Wageningen, pp. 8-23.

Marsden, T. K., 1998. New rural territories: regulating the differentiated rural spaces. Journal of Rural Studies 14(1), 107-117.

Midoux, B., 2013. Políticas para promover los circuitos cortos: la experiencia del Ministerio de Agricultura de Francia, in: Agricultura familiar y circuitos cortos. Nuevos esquemas de producción, comercialización y nutrición. CEPAL. Serie de Seminarios y Conferencias № 77, pp. 97-102.

Milone, P., 2013. Agricultura campesina y circuitos cortos: nuevas tendencias en Europa. En Agricultura familiar y circuitos cortos, in: Agricultura familiar y circuitos cortos. Nuevos esquemas de producción, comercialización y nutrición. CEPAL. Serie de Seminarios y Conferencias № 77, pp. 15-18.

Norgaard, R.B., 1994. Development Betrayed. The End of Progress and a Coevolutionary Revisioning of the Future. Routledge, New York.

Paz, R., 2008. Mitos y realidades sobre la agricultura familiar en Argentina en Problemas del Desarrollo. México. Revista Latinoamericana de Economía 39(153), 57-82.

Paz, R., Bruno S., 2013. El potencial de la agricultura familiar y los espacios protegidos: lineamientos para el diseño de políticas públicas. Revista Mundo Agrario 13(26).

Paz, R., Jara, C., Nazar, P., 2013. Economía Social y Agricultura Familiar. La experiencia de la Feria de Villa Río Hondo (Argentina), in: CAYAPA, Revista Venezolana de Economía Social. Año 13, № 25, pp. 53-73.

Paz, R., Rodríguez-Sperat, R., González, V., 2006. Desarrollo local y tipos de producciones. Revista Trabajo y Sociedad № 8 (VII).

Paz, R., Rodríguez-Sperat, R., González, V., Liphsitz, H., 2011. Producción económica en una pequeña explotación lechera caprina: hacia un diseño alternativo de desarrollo rural. Revista Asociación Latinoamericana de Producción Animal 18(3-4), 97-111.

Porter, M. E., 1996. What is Strategy? Harvard Business Review, Nov/Dec, 61-78.

Ramírez, E., Pino, R., Escobar, E., Quiroz, O., Ruiz, R., Sarmiento, R., Echeverría, J., 2007. Vinculación a mercados dinámicos de territorios rurales pobres y marginados. Editorial Fondo Mink'a de Chorlaví. < http://www.infoandina. org/node/19825>

Renting, H., Marsden, T. K., Banks, J., 2003. Understanding alternative food networks: exploring the role of short supply chains in rural development. Environment and planning $A$ 35, 393-411.

Rodríguez, F., 2013. La visión de las organizaciones de productores, in: Agricultura familiar y circuitos cortos. Nuevos esquemas de producción, comercialización y nutrición. CEPAL. Serie de Seminarios y Conferencias № 77, pp. 9-11.

Rodríguez-Sperat, R., Jara, C., Paz, R., 2014. Fortalezas y debilidades de los circuitos comerciales de la Agricultura familiar. Aportes para discutir sus potencialidades desde una experiencia en Santiago del Estero, in: Valenzuela, C., García, A. y Rosa, P. (Comp.), Inclusión social en las economías regionales. Estrategias participativas y propuestas de articulación social en el territorio. 1a ed. Instituto de Investigaciones Geohistóricas, pp. 35-58.

Rodríguez-Sperat, R., Peiretti, 0., 2007. El rol estratégico del 
gobierno provincial y las instituciones en el proceso de desarrollo territorial rural. El caso de la cuenca lechera caprina del área de riego de Santiago del Estero, in: Serie Estudios Sociales Agrarios - Sociología Rural: Doctorado en Estudios Sociales Agrarios. Córdoba, Argentina, pp. 107-134.

Schejtman, A., 1981. El agro mexicano y sus intérpretes. Revista Nexos № 39, 37-47.

Schejtman, A., 2008. Alcances sobre la Agricultura Familiar en América Latina, in: Diálogo Rural Iberoamericano, San Salvador. Documento de Trabajo $N^{\circ} 21$. Programa Dinámicas Territoriales Rurales. RIMISP, Santiago de Chile.

Schejtman, A., Ramírez, E., 2004. Desarrollo territorial rural. Aspectos destacados de experiencias en proceso en América Latina. Editado por Fondo Mink'a de Chorlaví. http://www.mapasderecursos.org.ar/archivos_biblioteca/1276009412_DTR_Fondo_Chorlavi_(1).pdf (acceso, 20.08.2014).

Shanin, T., 1973. The nature and logic of the peasant economy 1: A Generalisation 1. The Journal of Peasant Studies 1(1), 63-80.

Smith, G., 1984. Reflections on the social Relations of Simple Commodity Production. Journal of Peasant Studies 15(4), 500-529.

Toledo, V., 1990. The Ecological Rationality of Peasant Production, in: Altieri, M. Agroecology and Small Farm De- velopment. CRC Press, Ann Arbor, MI, pp. 51-58.

Van der Ploeg, J. D., 2007. 0 modo de produção camponês revisitado, in: "A diversidade da agricultura familiar" Schneider, S. (Comp.), Editora da UFRGS. Porto Alegre.

Van der Ploeg, J. D., 2010. Nuevos campesinos. Campesinos e Imperios Alimentarios. Icaria, Barcelona.

Van der Ploeg, J. D., 2013. Peasant and the art of farming. A Chayanovian manifesto. Agrarian change and peasant studies series. Fernwood Publishing, Halifax and Winnipeg.

Van der Ploeg, J. D., 2015. Newly emerging, nested markets. A theoretical introduction. The construction of new, nested markets and the role of rural development policies. Some introductory notes, in: Hebinck, P., Van der Ploeg, J.D., Schneider, S. (Eds.), Rural Development and the Construction of New Markets. Routledge, pp. 16-40.

Vasilachis de Gialdino, I., 2006. Estrategias de investigación cualitativa. Gedisa, Barcelona.

VSF-CICDA., 2013. Soberanía Alimentaria y comercialización campesina. Documento elaborado para la capacitación de Técnicos, Productores y Líderes Locales en fortalecimiento organizativo y comercialización campesina, en el marco del proyecto Mercados Campesinos. Quito, Ecuador. http://www.avsf.org/public/posts/1574/ boletin_1_formacion_lideres_soberania_alimentaria_ avsf_2013.pdf (acceso, 27.08.2014). 
